\title{
Deactivation routes in gold(I) polypyridyl complexes: Internal conversion vs Fast intersystem crossing
}

Elisabet Aguiló, ${ }^{\mathrm{a}}$ Artur J. Moro, ${ }^{\mathrm{b}}$ Mani Outis, ${ }^{\mathrm{b}}$ João Pina, ${ }^{\mathrm{c}}$ Daniela Sarmento, ${ }^{\mathrm{c}} \mathrm{J}$. Sérgio Seixas de Melo, ${ }^{\mathrm{c}}$ Laura Rodríguez, ${ }^{\mathrm{a}, \mathrm{d}}$ and João Carlos Lima. ${ }^{\mathrm{b}^{*}}$

${ }^{a}$ Departament de Química Inorgànica i Orgànica. Secció de Química Inorgànica. Universitat de Barcelona, Martí i Franquès 1-11, 08028 Barcelona, Spain.

${ }^{b}$ LAQV-REQUIMTE, Departamento de Química, CQFB, Universidade Nova de Lisboa, Monte de Caparica, Portugal.email:lima@fct.unl.pt

${ }^{c}$ Coimbra Chemistry Centre, Department of Chemistry, University of Coimbra, Rua Larga, 3004-535 Coimbra, Portugal

${ }^{d}$ Institut de Nanociència i Nanotecnologia (IN $\left.{ }^{2} U B\right)$. Universitat de Barcelona, 08028 Barcelona (Spain)

Dedicated to Prof. Fernando Pina on occasion of his $70^{\text {th }}$ anniversary. 


\begin{abstract}
An electronic spectral and photophysical characterization of three gold(I) complexes containing heterocyclic chromophores differing in the number and arrangement of pyridine rings (pyridine, bipyridine and terpyridine, with the acronyms $\mathrm{pD}, \mathrm{bD}$ and $\mathrm{tD}$ respectively) was performed. Quantum yields of fluorescence, internal conversion and triplet state formation, together with the rate constants for singlet to triplet intersystem crossing, $\mathrm{S}_{1} \sim \sim \mathrm{S}_{0}$ internal conversion and fluorescence were measured in order to equate the impact of fast triplet state formation on the amount of triplets formed. The results showed a correlation between the increase on the measured decay values of $S_{1}$ (leading to the main formation of $\mathrm{T}_{1}$ ) and the increase in the Charge Transfer (CT) character of the lowest energy transition, as evaluated from the orthogonality of the frontier orbitals. The measured triplet state quantum yields range from $\sim 50-60 \%$ to $70 \%$, while the intersystem crossing rate constants differ by almost 2 orders of magnitude, from $9.4 \times 10^{9} \mathrm{~s}^{-1}$ for tD to $8.1 \times 10^{11}$ $\mathrm{s}^{-1}$ for $\mathrm{bD}$. This constitutes an evidence for the existence of a correlation between the intersystem crossing and the internal conversion mechanisms.
\end{abstract}

Keywords: flash photolysis, gold(I), pump probe, luminescence 


\section{INTRODUCTION}

The design of compounds exhibiting high triplet yields continues to attract much interest mainly because of their potential ${ }^{1-7}$ in many different fields. In particular, gold(I) complexes, due to unique properties of gold, show potential uses as advanced materials, therapeutic drugs, photodynamic therapy agents and sensors, among others. ${ }^{8-12}$

Introduction of gold(I) cation can significantly modify the electronic states of an organic aromatic compound ${ }^{13-16}$ by enhancing spin-orbit coupling interactions, thus increasing the rate of radiationless transition between singlet and triplet states relative to the pure organic chromophore, ${ }^{16-19}$ as well as the radiative rates between the lowest triplet states and the singlet ground state. ${ }^{20}$ For instance, gold(I) complexes can exhibit dual luminescence at room temperature with emission yields that depend sensitively on the structure of the molecule. ${ }^{13,14,17,21-24}$

This makes gold(I) very appealing as an approach to the design of sensors, since due to the dual emission it can be exploited to reduce signal-to-noise ratio through a ratiometric response, thus independent of concentration. Secondly, since part of their luminescence results from triplet states, time-gated acquisition methods can be used, and these molecules display extremely high sensitivity to oxygen, temperature, $\mathrm{pH}$ and viscosity of the surrounding medium.

Information about the excited-state dynamics and relaxation mechanisms that are taking place in gold(I) complexes possessing different organic chromophores is essential for the rational design of advanced luminescent materials based on gold(I). ${ }^{12,17,18,25}$ Nevertheless, photophysical investigations performed with gold(I) complexes are focused mainly on describing qualitative data, e.g., the absorption and emission spectra, and not the quantitative data (quantum yields, decay times and rate constants for the deactivation processes), thus giving only a limited picture of the overall deactivation processes in these systems. Indeed, only a few of them are focused on the excited-state dynamics ${ }^{16,18}$ and point out some remarkably large intersystem rate constants $\left(10^{12}-10^{13} \mathrm{~s}^{-1}\right)$ which depend on the nature of the organic chromophore and the nature of the bonding to the gold atom. However, large intersystem crossing rate constants, do not necessarily allow high triplet 
yields and, simultaneous, measurements of both rate constants and yields for intersystem crossing of gold(I) complexes is absent in the literature.

Our recent research efforts have been turned to phosphine-Au(I)-akynyl complexes, ${ }^{26}$ due to their chemical versatility which allows modulation of solubility (phosphine ligand) ${ }^{27}$ and luminescence (ethynyl-organic chromophore ligand), conferring them with ideal properties for the development of new luminescent soft materials and sensing applications. ${ }^{28,29}$

In this work we present a systematic and comprehensive photophysical investigation of a series of ethynyl-gold complexes with different (poly)pyridyl chromophoric units (Chart 1) in which we analyze the intersystem crossing rates in solution and measure the yield values for triplet state formation. The results illustrate that high triplet yield values cannot be achieved simply by an increase in the intersystem crossing rate constants.
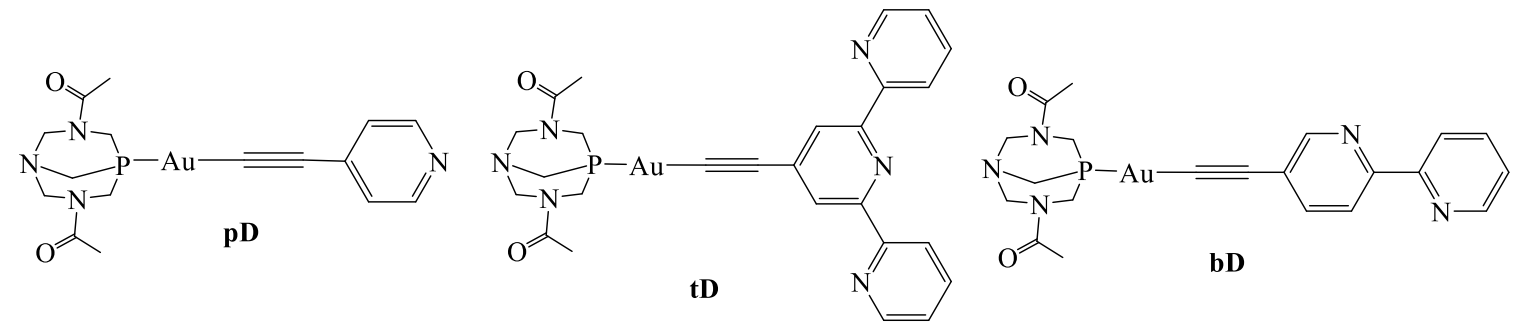

Chart 1. Structures and acronyms of the gold(I) compounds studied in this work. The first letter refers to the chromophore (" $p$ " for pyridine, " $t$ " for terpyridine and " $b$ " for bipyridine) and the second, D, refers to the DAPTA phosphine (3,7-diacetyl-1,3,7-triaza-5phosphabicyclo[3.3.1]nonane), common in all of them. 


\section{MATERIALS AND METHODS}

\section{General procedures}

Solvents and reagents - All solvents used were of spectroscopic grade. Acetonitrile was purchased from Sigma Aldrich. Deoxygenation was done by bubbling the solutions with a stream of argon during approximately $20 \mathrm{~min}$. Oxygenation was achieved by equilibration with air during $30 \mathrm{~min}$, following the spectral changes until a stationary state was achieved. All measured solutions were freshly prepared (within the day) unless noted otherwise.

Synthesis of the compounds - Literature methods have been used to prepare compounds $\mathrm{pD}^{30}, \mathrm{bD}^{29}$, and $\mathrm{tD} .^{29}$

\section{Physical measurements}

Absorption Spectroscopy - Absorption spectra were obtained in a $1 \mathrm{~cm}$ quartz cuvette in acetonitrile on a Varian Cary 100 Bio UV- spectrophotometer the concentrations used were $1.7 \cdot 10^{-5} \mathrm{M}(\mathrm{pD}), 2.1 \cdot 10^{-5} \mathrm{M}(\mathrm{bD})$ and $2.5 \cdot 10^{-5} \mathrm{M}(\mathrm{tD})$.

Emission spectroscopy - The emission spectra of oxygenated and deoxygenated solutions were obtained in fluorescence quartz cuvette $1 \mathrm{~cm}$, using a Horiba-Jobin-Yvon SPEX Nanolog spectrofluorimeter. The spectra were corrected for the photomultiplier response and the slits used were $4 \mathrm{~nm}(\mathrm{pD}), 2 \mathrm{~nm}(\mathrm{bD})$ and $3 \mathrm{~nm}(\mathrm{tD})$.

\section{Fluorescence quantum yield determination and Laser Flash Photolysis experiments}

All measured solutions were degassed using 3 freeze-pump-thaw cycles. Emission quantum yields were determined by employing tryptophan as a reference $\left(\Phi_{\mathrm{F}}=0.12,25^{\circ} \mathrm{C}\right.$, HEPES

buffer $0.05 \mathrm{M}$ at $\mathrm{pH}=7$ ), acquiring the spectra on a Horiba-Jobin-Yvon SPEX Fluorolog 3.22. Transient absorption spectra were measured using a flash photolysis setup composed of a LKS 60 ns laser photolysis spectrometer from Applied Photophysics, with a Brilliant Q-Switch Nd:YAG laser from Quantel, using the fourth harmonics $\left(\lambda_{\mathrm{ex}}=266 \mathrm{~nm}\right.$, laser pulse half-width equal to $6 \mathrm{~ns}$ ). 
The triplet yields $\left(\Phi_{\mathrm{T}}\right)$ of the compounds were measured by the energy transfer method ${ }^{31}$, equation 1, using $\beta$-carotene as triplet acceptor:

$$
\Phi_{\mathrm{T}}=\Phi_{\mathrm{T}}^{\mathrm{ref}} \times \frac{\Delta \mathrm{OD}_{\beta-\text { carotene }}^{\mathrm{CP}}}{\Delta \mathrm{OD}_{\beta-\text { carotene }}^{\mathrm{ref}}} \times \frac{\mathrm{P}_{\mathrm{et}}^{\mathrm{ref}}}{\mathrm{P}_{\mathrm{et}}^{\mathrm{CP}}}
$$

where ref stands for the reference solution containing 2-acetylnaphthalene $\left(\Phi_{\mathrm{T}}=0.84\right)^{32}$ in acetonitrile, optically matched with the sample solution at $266 \mathrm{~nm}$. $P_{e t}$ represents the energy transfer efficiency which was determined by equation 2 :

$$
P_{e t}=\frac{\tau-\tau \prime}{\tau}
$$

where $\tau$ is the lifetime of the donor in the absence of the acceptor and $\tau$ ' in the presence of the acceptor; this is done for the donors 2-acetylnaphthalene $\left(\mathrm{P}_{\mathrm{et}}^{\mathrm{ref}}\right)$ and the compounds studied $\left(\mathrm{P}_{\mathrm{et}}^{\mathrm{CP}}\right)$.

\section{Singlet oxygen yields}

Singlet oxygen yields were measured by direct measurement of the phosphorescence at $1270 \mathrm{~nm}$ followed upon irradiation of the aerated solution of the samples in ethanol with excitation at $266 \mathrm{~nm}$ from a Nd:YAG laser with a setup elsewhere described. ${ }^{33}$ Biphenyl in cyclohexane $\left(\phi_{\Delta}=0.73\right)$ was used as standard. ${ }^{34}$

\section{Femtosecond Transient Absorption Spectroscopy}

The ultrafast transient spectroscopic and kinetic measurements were performed in a Helios spectrometer (Ultrafast Systems) with an instrumental response function of around $250 \mathrm{fs}$ (apparatus described elsewhere). ${ }^{35}$ The instrumental response function of the system was assumed to be equal to that of the pump-probe cross correlation determined from the measurement of the instantaneous stimulated Raman signal from the pure solvent (in a 2 $\mathrm{mm}$ cuvette). To avoid photodegradation the solutions were stirred during the experiments or in movement using a motorized translating sample holder. The spectral chirp of the data was corrected using Surface Xplorer PRO program from Ultrafast Systems. Global analysis of the data (using a sequential model) was performed using Glotaran software. ${ }^{36}$ 


\section{TCSPC}

Fluorescence decays in aerated acetonitrile solutions were measured with the third harmonic of a Spectra Physics Tsunami Ti:Sa laser (excitation at $269 \mathrm{~nm}$ or $273 \mathrm{~nm}$ ) using a home-built time picosecond correlated single photon counting, TCSPC, apparatus (3 ps time resolution) described elsewhere. ${ }^{33}$ The fluorescence decays and the instrumental response function (FWHM) were collected with 4096 channels in an 814 fs/channel time scale. Deconvolution of the fluorescence decay curves was performed using the modulating function method, as implemented by G. Striker in the SAND program. ${ }^{37}$

\section{Molecular Orbitals}

DFT calculations have been carried out with the program Gaussian 09 using the PBE1PBE (PBE0) functional, 6-31G** basis set for $\mathrm{C}, \mathrm{H}, \mathrm{O}, \mathrm{N}$ and a standard double- $\zeta$ polarized basis set, namely, the LANL2DZ ${ }^{38}$ set augmented with polarization functions on $\mathrm{P}^{39}$ and $\mathrm{Au}$ (a $d$ orbital with exponent 0.364 for P, upon EMSL basis set exchange database) and an $f$ orbital with exponent 0.200 for $\mathrm{Au}) .{ }^{40}$ For $\mathrm{Au}$ and $\mathrm{P}$ were applied also Effective Core Potentials. PBE1PBE (PBE0) functional has been found to perform exceedingly well for many properties such as energetics, EPR properties, and specially excitation energies for transition metals $^{41,42}$ as well as in geometry predictions of Gold(I) complexes with unsaturated aliphatic hydrocarbons. ${ }^{43}$ Molecular Orbital visualisation for all the complexes was performed using Avogadro 1.2.0. 


\section{RESULTS}

\section{Absorption and emission with and without oxygen}

The absorption and emission spectra of the complexes in freshly prepared acetonitrile solutions are presented in Figure 1. Comparison of the emission of deaerated (by bubbling $\mathrm{N}_{2}$ ) and aerated solutions is also presented. The absorption bands present the typical features of the $\pi \pi^{*}$ transition present in the aromatic ligand. ${ }^{26,28,29,44-46}$

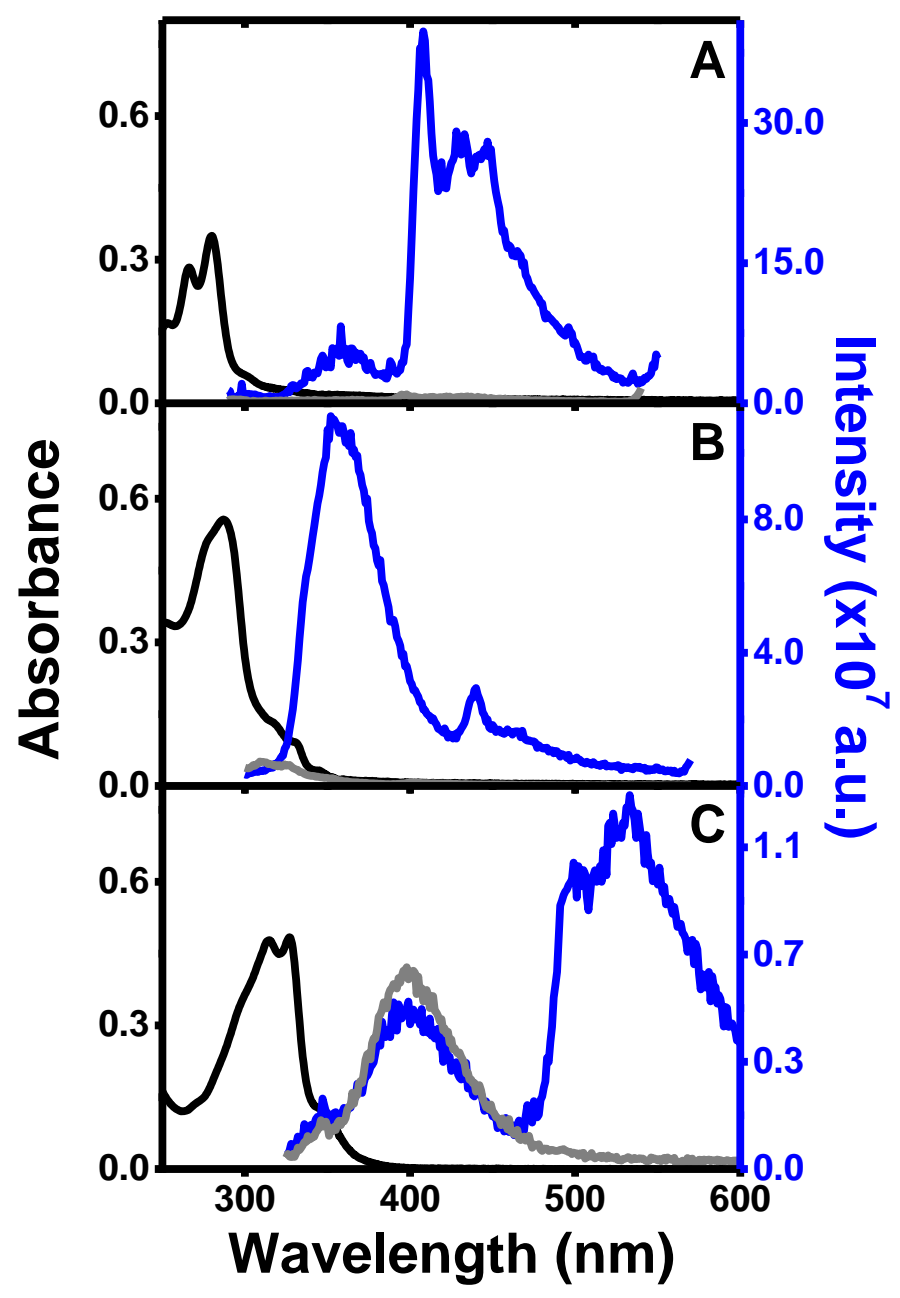

Figure 1. Absorption (black line), emission in the presence of oxygen (gray line) and in deaerated solutions (blue line) of (A) pD $1.7 \times 10^{-5} \mathrm{M}, \lambda_{\text {exc }}=280 \mathrm{~nm}$; (B) tD $2.5 \times 10^{-5} \mathrm{M}$,

$$
\lambda_{\mathrm{exc}}=290 \mathrm{~nm} ;(\mathrm{C}) \mathrm{bD} 2.1 \times 10^{-5} \mathrm{M}, \lambda_{\mathrm{exc}}=315 \mathrm{~nm} \text { in acetonitrile. }
$$

In all cases the emission is composed by two bands, the highest energy band presents a small Stokes shift with respect to the absorption band and is assigned to radiative emission 
(fluorescence) from the $S_{1}$ state, while the lowest energy band, with a significant Stokes shift, observed only after oxygen removal, is assigned to phosphorescence (from the $\mathrm{T}_{1}$ state).

Table 1 - Lowest singlet, $\mathrm{E}_{\mathrm{S} 1}$ and triplet, $\mathrm{E}_{\mathrm{T} 1}$, energies, Quantum Yield of fluorescence, $\Phi_{\mathrm{f}}$, triplet formation, $\Phi_{\mathrm{T}}$, and ${ }^{1} \mathrm{O}_{2}$ formation, $\Phi_{\Delta}$, triplet decay time in the absence of oxygen, $\tau_{\mathrm{T} 1}$, measured for complexes $\mathrm{pD}, \mathrm{tD}$ and $\mathrm{bD}$ in acetonitrile.

\begin{tabular}{|l|l|l|l|l|l|l|}
\hline Compound & $\mathrm{E}_{\mathrm{S} 1}(\mathrm{eV})$ & $\mathrm{E}_{\mathrm{T} 1}(\mathrm{eV})$ & $\Phi_{\mathrm{f}} *$ & $\Phi_{\mathrm{T}}$ & $\Phi_{\Delta} *$ & $\begin{array}{l}\tau_{\mathrm{T} 1} \\
(\mu \mathrm{s})\end{array}$ \\
\hline $\mathrm{bD}$ & 3.6 & 2.7 & 0.001 & 0.70 & 0.33 & 70 \\
\hline $\mathrm{pD}$ & 3.9 & 3.2 & 0.001 & 0.53 & 0.16 & 15 \\
\hline $\mathrm{tD}$ & 3.8 & 3.0 & 0.005 & 0.63 & 0.39 & 38 \\
\hline
\end{tabular}

The effect of oxygen removal leads to distinct effects on the intensity of phosphorescence and fluorescence bands. In the case of the phosphorescence band all the compounds show a significant increase in phosphorescence intensity. This is expected for triplets of energies above $95 \mathrm{~kJ} \mathrm{~mol}^{-1}(0.98 \mathrm{eV})$, possessing lifetimes higher than $1 \mu \mathrm{s}$, which are known to transfer energy to the ground state triplet oxygen molecule and produce singlet oxygen. ${ }^{47}$ The measured singlet oxygen quantum yields are presented in Table 1, together with $\phi_{\mathrm{T}}$ values and triplet decay times (degassed solutions) obtained from laser flash photolysis experiments (Figure S1 in SI).

Compounds $\mathrm{pD}$ and $\mathrm{tD}$ also show enhancement of the fluorescence band upon oxygen removal. This can be assigned to triplet-triplet annihilation leading to different amounts of delayed fluorescence in the different compounds. We have previously shown, for several gold(I) alkynyl complexes, that they aggregate through the establishment of metallophilic interactions, leading to nanometer size aggregates. ${ }^{29}$ Triplet-triplet annihilation is likely to occur inside the aggregates. This was corroborated by the fact that equilibrium with oxygen from air immediately quenches the phosphorescence band, while the quenching of the fluorescence band takes several minutes (results not shown). Additionally, the fluorescence 
emission of $\mathrm{tD}$ is observed to increase with time upon formation of aggregates (see figure $\mathrm{S} 2$ in SI).

In all cases the triplet formation quantum yield is not unitary. As expected the $\Phi_{\Delta}$ values (Table 1) are lower than the measured triplet formation quantum yields, $\Phi_{\mathrm{T} .}{ }^{47}$

\section{Broadband Transient Absorption Experiments.}

The transient absorption map of bD in acetonitrile (pumped at $330 \mathrm{~nm}$ ) shows only the presence of a single relaxation at very short times (Figures 2 and S3). Global analysis performed in Glotaran ${ }^{36}$ with a sequential model fits all data points with an exponential decay with a very short decay time (860 fs) and a second (fixed) decay component of $33 \mu \mathrm{s}$ describing the $T_{1}$ final state which does not decay in this time scale. The initial state, assigned to a relaxed singlet state $\left(\mathrm{S}_{1}\right.$ or $\left.{ }^{1} \mathrm{CT}\right)$, relaxes in 860 fs to yield the final $\mathrm{T}_{1}$ state (see below assignment of $\mathrm{T}_{1}$ ).

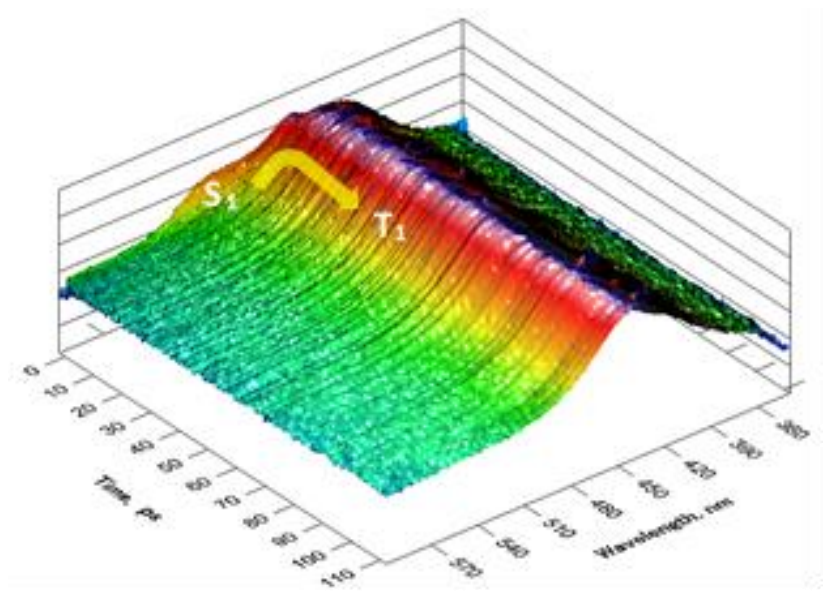

Figure 2 - Transient absorption map of bD in acetonitrile, pumped with $330 \mathrm{~nm}$ excitation.

Similar analysis with pD and tD (Figures S4 and S5) shows that the $\mathrm{T}_{1}$ state is formed much slower (14.6 ps and 66.9 ps for $\mathrm{pD}$ and $\mathrm{tD}$ respectively) than in the case of $\mathrm{bD}$. Furthermore, the rates of $\mathrm{T}_{1}$ formation (see kISC in Table 2 below) do not follow the order of the triplet yields measured (Table 1).

Transient absorption spectra at selected time delays are given in Figure 3 for compounds $\mathrm{bD}, \mathrm{pD}$ and $\mathrm{tD}$ in acetonitrile, together with the fitted time traces at the maxima of the observed transient absorption bands. 

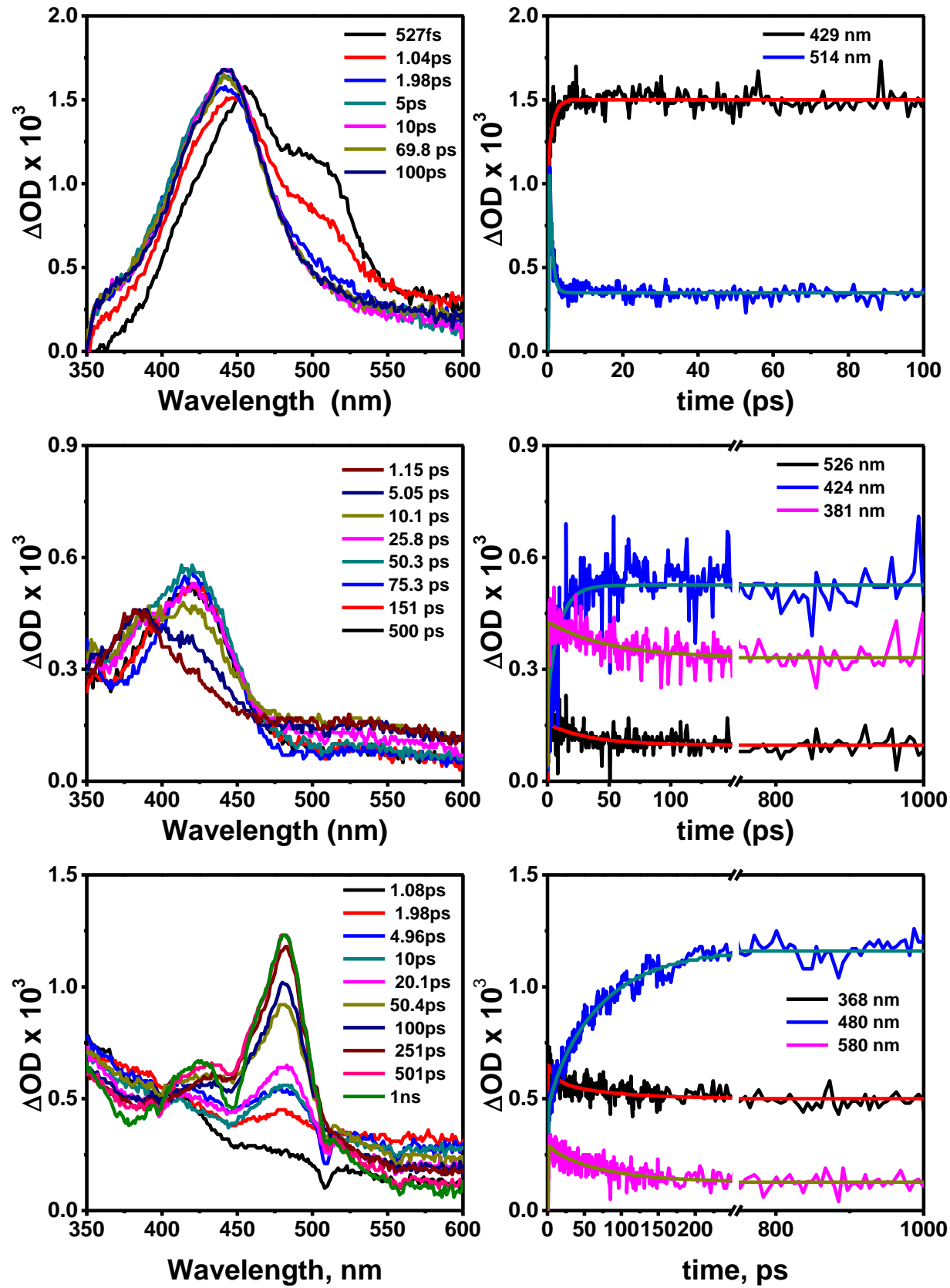

Figure 3. Transient absorption spectra at selected time delays (left), time evolution of the transients, fitted with single exponential laws (right) for acetonitrile solutions of bD (top), $\mathrm{pD}$ (middle) and tD (bottom), at room temperature.

The presence of isosbestic points and the quality of the single exponential fittings, clearly corroborates a two state relaxation process, where the initial state, populated by the 
pumping event and assigned to $\mathrm{S}_{1}$ or ${ }^{1} \mathrm{CT}$, relaxes to a final state assigned to $\mathrm{T}_{1}$ (see below); the $\mathrm{T}_{1}$ triplet does not decay in the time window of the fs-TA pump-probe experiment, but is observed and defined in a longer time-scale (see next section).

\section{Nanosecond to microsecond transient absorption}

Nanosecond laser flash photolysis with excitation at $266 \mathrm{~nm}$ was used to collect the transients in the microsecond time scale. Figure 4 shows the typical transient absorption of the compounds (blue diamonds) in the microsecond time window superimposed with the initial and final transients obtained from the fs-TA experiments in Figure 3.

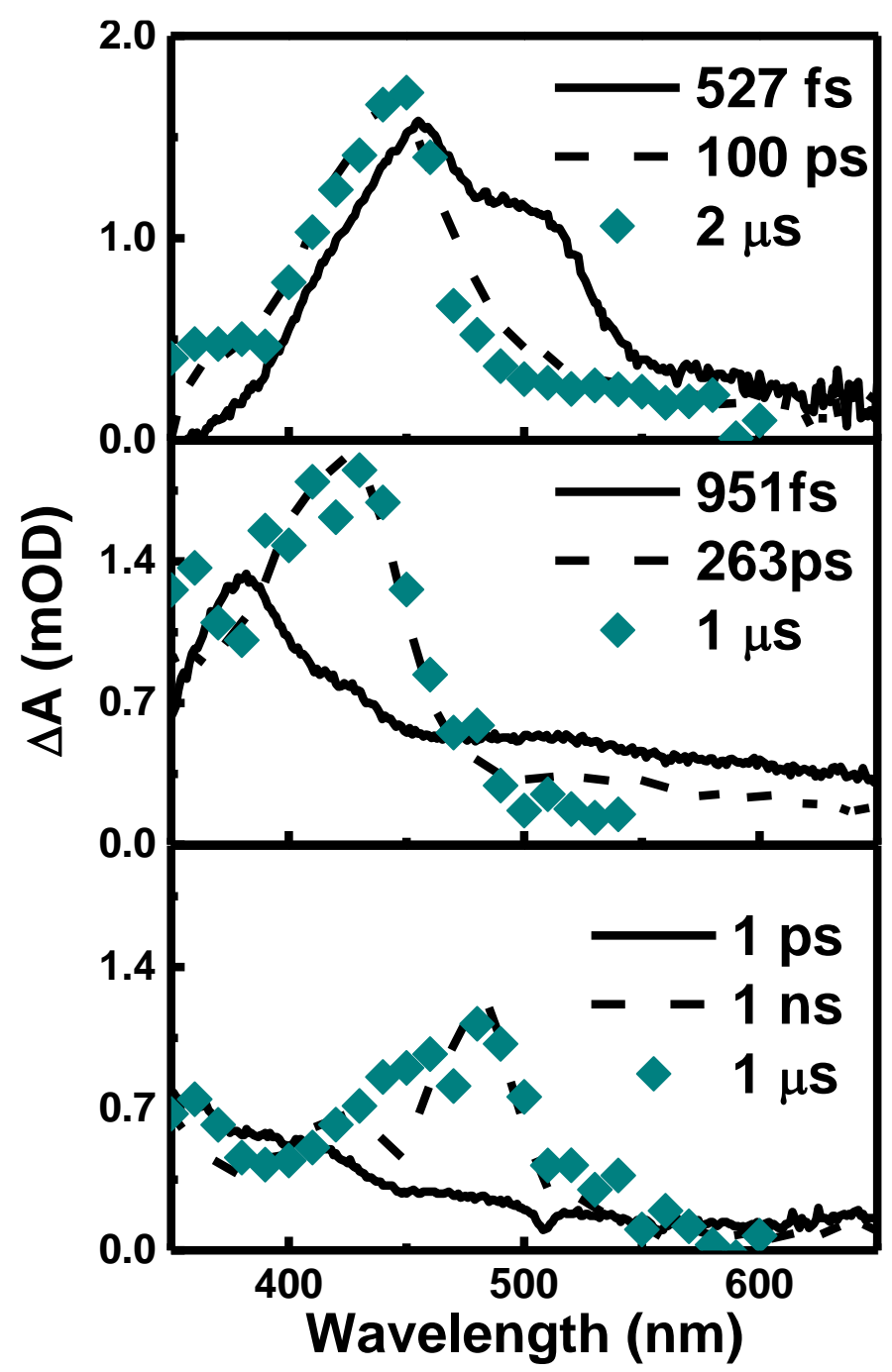

Figure 4 - Nanosecond laser flash photolysis transient absorption spectra with excitation at $266 \mathrm{~nm}$ of $\mathrm{bD}$ (top), $\mathrm{pD}$ (middle) and $\mathrm{tD}$ (bottom) obtained in the $\mu$ s time scale (blue 
diamonds) superimposed to the femtosecond transients collected at short time after pump (solid line) and after stabilization of the absorption (dashed line).

As can be observed from Figure 4, there is an excellent superposition between the transient absorption obtained at longer times in the femtosecond transient absorption measurements and the transient absorption collected with laser flash photolysis at microsecond time scales (Figure 4). The transients observed in the microsecond time scale decay with single exponential decay laws (lifetimes are collected in Table 1 and decays displayed in Figure S1) are fitted with identical lifetimes to the recovery of the ground state bleaching. These observations, together with the observation of quenching of the microsecond decay times by oxygen, allow the assignment of the transient absorptions as $T_{1}-T_{n}$ transitions, and consequently that the final state of the femtosecond transient absorption experiment is, in all cases, the $\mathrm{T}_{1}$ state. Noteworthy, contrary to the behavior found for the $\mathrm{pD}$ and $\mathrm{tD}$ derivatives, the bD characteristic triplet transient absorption band is blue-shifted with respect to the $S_{1}-S_{n}$ band. Indeed, similar behavior was previously reported for 2,2'bipyridine thus giving support to the assignment of the time resolved spectra collected in the 0.527-1.04 ps range (Figure 3 and 4 ) to the precursor of the $T_{1}$ state, i.e. the $S_{1}$ state. ${ }^{48}$

\section{DISCUSSION}

It was previously reported for a series of phosphine-gold(I)-naphthalene derivatives the presence of very fast intersystem crossing rate constants $\left(10^{-12}-10^{-13} \mathrm{~s}^{-1}\right)$ and a slower relaxation $\mathrm{T}_{\mathrm{n}}-\mathrm{T}_{1}(\sim 10 \mathrm{ps})$ process. ${ }^{16}$ The occurrence of intersystem crossing within the femtosecond pulse ( 250 fs) cannot be totally discarded, although for the present investigated compounds there is some data that makes this hypothesis less probable. Indeed, the fluorescence decays for all the complexes were collected along the fluorescence emission band (Figure 5 and Table 2), to rule out the possibility of a faster (sub picosecond) $\mathrm{S}_{1}$ relaxation. 
In the absence of contradicting evidence we will consider reasonable to assign $S_{1}$ as the initially observed state, i.e., we have found no evidence of a slow picosecond $T_{n}-T_{1}$ relaxation, preceded by a very fast femtosecond intersystem crossing process. However, we cannot discard that this $\mathrm{S}_{1}$ state could be a relaxed ${ }^{1} \mathrm{CT}$ state, and that the conversion between the locally excited singlet state (formed by the pumping) and the charge transfer state occurs faster than our experimental resolution. Table 2 summarizes the internal conversion, $\mathrm{k}_{\mathrm{IC}}$, intersystem crossing, $\mathrm{k}_{\mathrm{ISC}}$, and radiative rate constants, $\mathrm{k}_{\mathrm{F}}$, for the $\mathrm{S}_{1}$ state of the studied complexes, obtained from the measured fluorescence and triplet state formation quantum yields and singlet state decay times.

Table 2. Decay time of the lowest lying singlet excited state $S_{1}, \tau_{S 1}$, quantum yields of intersystem crossing, $\Phi_{\mathrm{T}}$, fluorescence, $\Phi_{\mathrm{f}}$, and internal conversion, $\Phi_{\mathrm{IC}}$ and rate constants of intersystem crossing, $\mathrm{k}_{\mathrm{ISC}}$, radiative, $\mathrm{k}_{\mathrm{F}}$, and internal conversion, $\mathrm{k}_{\mathrm{IC}}$, of the singlet state.

\begin{tabular}{|l|l|l|l|l|l|l|l|}
\hline Compound & $\begin{array}{l}\tau_{\mathbf{S} 1} \\
(\mathbf{p s})\end{array}$ & $\boldsymbol{\Phi}_{\mathbf{T}}$ & $\boldsymbol{\Phi}_{\mathbf{f}}$ & $\boldsymbol{\Phi}_{\mathbf{I C}}$ & $\begin{array}{l}\mathbf{k}_{\mathbf{I S C}} \\
\left(\mathbf{s}^{\mathbf{- 1}}\right)\end{array}$ & $\begin{array}{l}\mathbf{k}_{\mathbf{F}} \\
\left(\mathbf{s}^{-\mathbf{1}}\right)\end{array}$ & $\begin{array}{l}\mathbf{k}_{\mathbf{I C}} \\
\left(\mathbf{s}^{-\mathbf{1}}\right)\end{array}$ \\
\hline bD & 0.86 & 0.70 & 0.001 & 0.30 & $8.1 \times 10^{11}$ & $1.2 \times 10^{9}$ & $3.5 \times 10^{11}$ \\
\hline pD & 14.6 & 0.53 & 0.001 & 0.47 & $3.6 \times 10^{10}$ & $6.9 \times 10^{7}$ & $3.2 \times 10^{10}$ \\
\hline tD & 66.9 & 0.63 & 0.005 & 0.37 & $9.4 \times 10^{9}$ & $7.5 \times 10^{7}$ & $5.5 \times 10^{9}$ \\
\hline
\end{tabular}

From Table 2 it can be seen that the increase in the intersystem crossing rate from $S_{1}$ to $T_{1}$ is accompanied by a simultaneous increase in the internal conversion from $\mathrm{S}_{1}$ to $\mathrm{S}_{0}$. This relation was already observed in organic compounds with electronic transitions with significant charge transfer character. ${ }^{49}$

In previously reported gold(I) complexes with pyrene and naphthalene chromophoric units, the $S_{1}$ and $T_{1}$ states were identified as mainly ligand centered (LC), perturbed by the heavy atom effect of gold. ${ }^{16,18}$ The inclusion of a heteroatom, as in the present case, clearly confers a strong intramolecular charge transfer character to the transitions (Figure 6) and could be responsible for the association, i.e., close proximity of the $\mathrm{k}_{\mathrm{ISC}}$ and $\mathrm{k}_{\mathrm{IC}}$ values. 

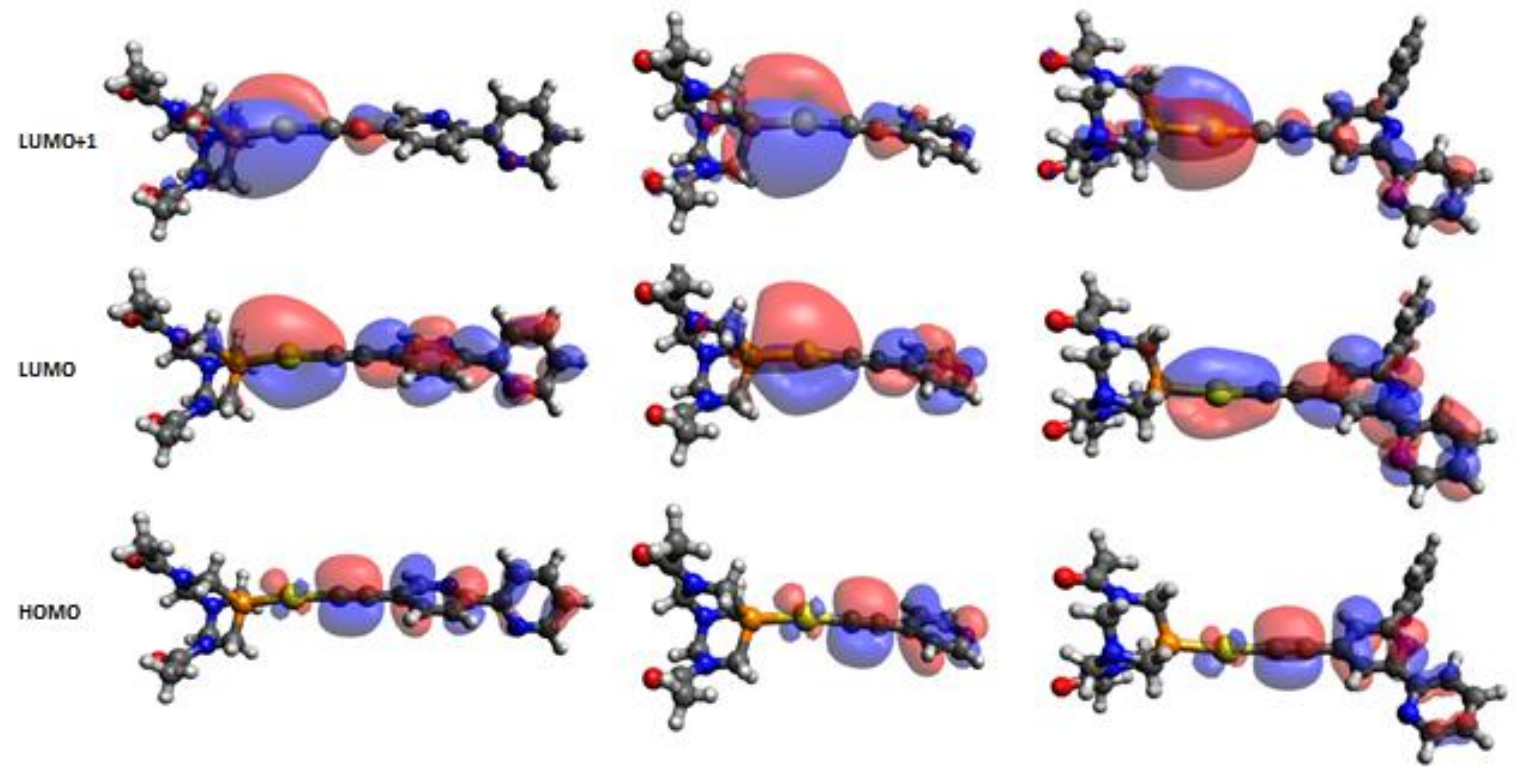

Figure 6. Frontier orbitals of the complexes illustrating the charge transfer of the HOMOLUMO transitions and HOMO-LUMO+1 transitions.

The frontier orbitals of the complexes involved in the lowest energy transitions (HOMO, LUMO and LUMO+1) were calculated (Figure 6 and Table S4) with the program Gaussian 09 using the PBE1PBE (PBE0) functional, 6-31G** basis set for $\mathrm{C}, \mathrm{H}, \mathrm{O}, \mathrm{N}$ and a standard double- $\zeta$ polarized basis set, namely, the set augmented with polarization functions on $\mathrm{P}^{39}$ and $\mathrm{Au}$ (a $d$ orbital with exponent 0.364 for $\mathrm{P}$, upon EMSL basis set exchange database) and an $f$ orbital with exponent 0.200 for $\mathrm{Au}) .{ }^{40}$ For $\mathrm{Au}$ and $\mathrm{P}$ were applied also Effective Core Potentials. The lowest singlet and triplet transitions calculated using TDDFT are presented in supplementary information. The complexes have very similar density distributions, and the intramolecular charge transfer character is evident upon promoting an electron from either HOMO to LUMO (more allowed singlet transition) or HOMO to LUMO+1 (transition with stronger CT character). The bD complex shows the larger orthogonality between initial and final state (stronger CT character), in particular between HOMO and LUMO+1 (Figure 6 and Table S4), which is amplified by the linear 
arrangement of the pyridine chromophores (longer distance from the second pyridine ring to gold).

Besides the heavy atom effect, the charge transfer state is probably intermediating the intersystem crossing, as previously shown for other molecules. ${ }^{50}$ Furthermore, increased orthogonality between the HOMO and the LUMO in CT states is known to decrease the energy splitting between singlet $\left({ }^{1} \mathrm{CT}\right)$ and triplet $\left({ }^{3} \mathrm{CT}\right)$ states leading to enhanced intersystem crossing upon mixing with other localized triplets of adequate simmetry. ${ }^{51}$

The relation between intersystem crossing and internal conversion rate constants was previously illustrated for organic chromophores, ${ }^{49}$ but in the case of gold(I) complexes we could not find published works that simultaneously measure intersystem crossing rates and triplet state quantum yields, in order to evaluate if this relation is part of a generalized mechanism. Those measurements are important to correct the misleading concept that very fast intersystem crossing rates necessarily lead to triplet formation quantum yields close to unit.

As it can be seen in the present case, the intersystem crossing quantum yields (see Table 2) are moderate, even for the highest intersystem crossing rate constant, $3.1 \times 10^{11} \mathrm{~s}^{-1}$, obtained for $\mathrm{bD}$. The reason for the observed behavior is that the values for internal conversion from S1 to the ground state are of the same order of magnitude as the intersystem crossing. 


\section{Conclusions}

The analysis of the photophysical data retrieved by different time-resolved spectroscopic techniques allows us to detect fast intersystem crossing rates in our series of gold(I) complexes. These rate constants are observed to be strongly dependent on the chromophore structure.

Gold(I) complexes tune effectively the excited-state dynamics of the organic compounds opening a fast channel for population of the phosphorescent states in these complexes. However, this is not associated with unity quantum yields of triplet formation, at least in solution, since the internal conversion from the singlet excited state is a deactivation channel competitive with intersystem crossing.

The presence of isosbestic points in the broadband transient absorption experiments and the quality of single exponential fittings, corroborate a simple two state relaxation process, where the initial state populated by the pumping event, assigned to $S_{1}$, relaxes to a final state assigned to $T_{1}$, which does not decay in the time window of the pump-probe experiment.

Time resolved fluorescence measurements yield decay times that are in reasonable agreement with the observed decay observed in pump probe experiments. We therefore found no evidence of a slow picosecond $\mathrm{Tn}-\mathrm{T} 1$ relaxation, preceded by a very fast femtosecond intersystem crossing process in this family of complexes. 


\section{Supporting Information}

Laser flash photolysis transients of optically matched solutions at $266 \mathrm{~nm}$ of bD, pD and tD in acetonitrile (Figure S1). Fluorescence emission spectra for deareated tD sample in acetonitrile excited at $280 \mathrm{~nm}$ as a function of time (Figure S2); Transient absorption map of bD in acetonitrile, pumped with $330 \mathrm{~nm}$ excitation (Figure S3); Transient absorption map of $\mathrm{pD}$ in acetonitrile, pumped with $280 \mathrm{~nm}$ excitation (Figure S4); Transient absorption map of tD in acetonitrile, pumped with $280 \mathrm{~nm}$ excitation (Figure S5); Z-matrix for bD (Table S1); Z-matrix for pD (Table S2); Z-matrix for tD (Table S3) and TDDFT electronic transitions (Table S4).

\section{Acknowledgements}

The research leading to these results has received funding from Laserlab-Europe (grant agreement no. 284464, EC's Seventh Framework Programme). The authors are grateful to the Ministry of Economy, Industry and Competitiveness of Spain (AEI/FEDER, UE Project CTQ2016-76120-P). This work was also supported by the Associated Laboratory for Sustainable Chemistry, Clean Processes and Technologies, LAQV, which is financed by national funds from FCT/MEC (UID/QUI/50006/2013) and co-financed by the ERDF under the PT2020 Partnership Agreement (POCI-01-0145-FEDER-007265). We also acknowledge funding from FEDER (Fundo Europeu de Desenvolvimento Regional) through COMPETE (Programa Operacional Factores de Competitividade). The Coimbra Chemistry Centre is supported by the Fundação para a Ciência e a Tecnologia (FCT), Portuguese Agency for Scientific Research, through the programme UID/QUI/UI0313/2013. J. Pina acknowledges the project SunStorage - Harvesting and storage of solar energy", for financial support, reference POCI-01-0145-FEDER-016387, funded by European Regional Development Fund (ERDF), through COMPETE 2020 - Operational Programme for Competitiveness and Internationalization (OPCI), and by national funds, through FCT. 


\section{References}

(1) Pollum, M.; Jockusch, S.; Crespo-Hernández, C. E. 2,4-Dithiothymine as a Potent UVA Chemotherapeutic Agent. J. Am. Chem. Soc. 2014, 136 (52), 17930-17933.

(2) Reichardt, C.; Crespo-Hernaíndez, C. E. Room-Temperature Phosphorescence of the DNA Monomer Analogue 4-Thiothymidine in Aqueous Solutions after UVA Excitation. J. Phys. Chem. Lett. 2010, 1 (15), 2239-2243.

(3) Allendorf, M. D.; Bauer, C. A.; Bhakta, R. K.; Houk, R. J. T. Luminescent Metalorganic Frameworks. Chem. Soc. Rev. 2009, 38 (5), 1330.

(4) Segura Carretero, A.; Salinas Castillo, A.; Fernández Gutiérrez, A. A Review of Heavy-Atom-Induced Room-Temperature Phosphorescence: A Straightforward Phosphorimetric Method. Crit. Rev. Anal. Chem. 2005, 35 (1), 3-14.

(5) Yersin, H. Highly Efficient OLEDs with Phosphorescent Materials; Yersin, H., Ed.; Wiley-VCH Verlag GmbH \& Co. KGaA: Weinheim, Germany, 2007.

(6) Duncan, T. V.; Frail, P. R.; Miloradovic, I. R.; Therien, M. J. Excitation of Highly Conjugated (Porphinato)Palladium(II) and (Porphinato)Platinum(II) Oligomers Produces Long-Lived, Triplet States at Unit Quantum Yield That Absorb Strongly over Broad Spectral Domains of the NIR. J. Phys. Chem. B 2010, 114 (45), 1469614702.

(7) Celli, J. P.; Spring, B. Q.; Rizvi, I.; Evans, C. L.; Samkoe, K. S.; Verma, S.; Pogue, B. W.; Hasan, T. Imaging and Photodynamic Therapy: Mechanisms, Monitoring, and Optimization. Chem. Rev. 2010, 110 (5), 2795-2838.

(8) Gao, L.; Niedzwiecki, D. S.; Deligonul, N.; Zeller, M.; Hunter, A. D.; Gray, T. G. Gold(I) Styrylbenzene, Distyrylbenzene, and Distyrylnaphthalene Complexes: High Emission Quantum Yields at Room Temperature. Chem. - A Eur. J. 2012, 18 (20), 6316-6327.

(9) Craig, S.; Gao, L.; Lee, I.; Gray, T.; Berdis, A. J. Gold-Containing Indoles as Anticancer Agents That Potentiate the Cytotoxic Effects of Ionizing Radiation. $J$. Med. Chem. 2012, 55 (5), 2437-2451.

(10) Partyka, D. V.; Zeller, M.; Hunter, A. D.; Gray, T. G. Relativistic Functional 
Groups: Aryl Carbon-Gold Bond Formation by Selective Transmetalation of Boronic Acids. Angew. Chemie - Int. Ed. 2006, 45 (48), 8188-8191.

(11) Gielen, M.; Tiekink, E. R. T. Metallotherapeutic Drugs and Metal-Based Diagnostic Agents: The Use of Metals in Medicine; 2005.

(12) Carlos Lima, J.; Rodríguez, L. Applications of Gold(i) Alkynyl Systems: A Growing Field to Explore. Chem. Soc. Rev. 2011, 40 (11), 5442-5456.

(13) Partyka, D. V.; Esswein, A. J.; Zeller, M.; Hunter, A. D.; Gray, T. G. Gold(I) Pyrenyls: Excited-State Consequences of Carbon-Gold Bond Formation.

Organometallics 2007, 26 (14), 3279-3282.

(14) Heng, W. Y.; Hu, J.; Yip, J. H. K. Attaching Gold and Platinum to the Rim of Pyrene: A Synthetic and Spectroscopic Study. Organometallics 2007, 26 (27), 67606768.

(15) Gao, L.; Peay, M. A.; Partyka, D. V.; Updegraff, J. B.; Teets, T. S.; Esswein, A. J.; Zeller, M.; Hunter, A. D.; Gray, T. G. Mono- and Di-Gold(I) Naphthalenes and Pyrenes: Syntheses, Crystal Structures, and Photophysics. Organometallics 2009, 28 (19), 5669-5681.

(16) Vogt, R. A.; Gray, T. G.; Crespo-Hernández, C. E. Subpicosecond Intersystem Crossing in Mono- and Di(Organophosphine)Gold(I) Naphthalene Derivatives in Solution. J. Am. Chem. Soc. 2012, 134 (36), 14808-14817.

(17) Lu, W.; Kwok, W. M.; Ma, C.; Chan, C. T. L.; Zhu, M. X.; Che, C. M. Organic Triplet Excited States of Gold(I) Complexes with Oligo(o-or mPhenyleneethynylene) Ligands: Conjunction of Steady-State and Time-Resolved Spectroscopic Studies on Exciton Delocalization and Emission Pathways. J. Am. Chem. Soc. 2011, 133 (35), 14120-14135.

(18) Vogt, R. A.; Peay, M. A.; Gray, T. G.; Crespo-Hernández, C. E. Excited-State Dynamics of ( Organophosphine ) Gold ( I ) Pyrenyl Isomers. J. Phys. Chem. Lett. 2010, 1205-1211.

(19) Rodríguez, L.; Lima, J. C.; Ferrer, M.; Rossell, O.; Engeser, M. 3D Au-Ag Heterometallic Supramolecular Cage: Triplet Capture by Heavy Atom Effect. Inorganica Chim. Acta 2012, 381 (1), 195-202.

(20) Rodríguez, L.; Ferrer, M.; Crehuet, R.; Anglada, J.; Lima, J. C. Correlation between 
Photophysical Parameters and Gold-Gold Distances in Gold(I) (4-Pyridyl)Ethynyl Complexes. Inorg. Chem. 2012, 51 (14), 7636-7641.

(21) Gao, L.; Partyka, D. V; Updegraff, J. B.; Deligonul, N.; Gray, T. G. Synthesis, Structures, and Excited-State Geometries of Alkynylgold(I) Complexes. Eur. J. Inorg. Chem. 2009, 2009 (18), 2711-2719.

(22) Chao, H.; Lu, W.; Li, Y.; Chan, M. C. W.; Che, C.; Cheung, K.; Zhu, N. Organic Triplet Emissions of Arylacetylide Moieties Harnessed through Coordination to $[\mathrm{Au}(\mathrm{PCy} 3)]+$. Effect of Molecular Structure upon Photoluminescent Properties. $J$. Am. Chem. Soc. 2002, 124 (49), 14696-14706.

(23) Peay, M. A.; Heckler, J. E.; Deligonul, N.; Gray, T. G. Red-Shifts upon Metal Binding: A Di-Gold(I)-Substituted Bithiophene. Organometallics 2011, 30 (18), 5071-5074.

(24) Gavara, R.; Aguiló, E.; Schur, J.; Llorca, J.; Ott, I.; Rodríguez, L. Inorganica Chimica Acta Study of the Effect of the Chromophore and Nuclearity on the Aggregation and Potential Biological Activity of Gold ( I ) Alkynyl Complexes. Inorganica Chim. Acta 2016, 446, 189-197.

(25) Raubenheimer, H. G.; Schmidbaur, H. Gold Chemistry Guided by the Isolobality Concept. Organometallics 2012, 31 (7), 2507-2522.

(26) Gavara, R.; Llorca, J.; Lima, J. C.; Rodríguez, L. A Luminescent Hydrogel Based on a New Au(I) Complex. Chem. Commun. 2013, 49 (1), 72-74.

(27) Darensbourg, D. J.; Ortiz, C. G.; Kamplain, J. W. A New Water-Soluble Phosphine Derived from 1,3,5-Triaza-7-Phosphaadamantane (PTA), 3,7-Diacetyl-1,3,7-Triaza5-Phosphabicyclo[3.3.1]Nonane. Structural, Bonding, and Solubility Properties. Organometallics 2004, 23 (8), 1747-1754.

(28) Ferrer, M.; Giménez, L.; Gutiérrez, A.; Lima, J. C.; Martínez, M.; Rodríguez, L.; Martín, A.; Puttreddy, R.; Rissanen, K. Polypyridyl-Functionalizated Alkynyl Gold(I) Metallaligands Supported by Tri- and Tetradentate Phosphanes. Dalton Trans. 2017, 46 (40), 13920-13934.

(29) Aguiló, E.; Moro, A. J.; Gavara, R.; Alfonso, I.; Pérez, Y.; Zaccaria, F.; Guerra, C. F.; Malfois, M.; Baucells, C.; Ferrer, M.; et al. Reversible Self-Assembly of WaterSoluble Gold(I) Complexes. Inorg. Chem. 2018, 57 (3), 1017-1028. 
(30) Aguiló, E.; Gavara, R.; Lima, J. C.; Llorca, J.; Rodríguez, L. From Au(i)

Organometallic Hydrogels to Well-Defined Au(0) Nanoparticles. J. Mater. Chem. C 2013, $1,5538-5547$.

(31) Elisei, F.; Lima, J. C.; Ortica, F.; Aloisi, G. G.; Costa, M.; Leitão, E.; Abreu, I.; Dias, A.; Bonifácio, V.; Medeiros, J.; et al. Photophysical Properties of HydroxySubstituted Flavothiones. J. Phys. Chem. A 2000, 104 (25), 6095-6102.

(32) Montalti, M.; Credi, A.; Prodi, L.; Gandolfi, M. T. Handbook of Third Edition; 2006.

(33) J.S. Seixas de Melo, J. Pina, F.B. Dias, A.L. Maçanita, Experimental Techniques for Excited State Characterisation, in: R.C. Evans, P. Douglas, H.D. Burrows (Eds.) Applied Photochemistry, Springer Netherlands 2013, pp. 533-585.

(34) Kristiansen, M., Scurlock, R.D., Iu, K-K., Ogilby, P.R. Charge-Transfer State and Singlet Oxygen Production in Photoexcited Organic Molecule-Molecular Oxygen Complexes. J. Phys. Chem. 1991, 95 (7), 5190-5197.

(35) Pina, J.; Seixas de Melo, J. S.; Eckert, A.; Scherf, U. Unusual Photophysical Properties of Conjugated, Alternating Indigo-fluorene Copolymers. J. Mater. Chem. A 2015, 3 (12), 6373-6382.

(36) Snellenburg, J. J.; Laptenok, S. P.; Seger, R.; Mullen, K. M.; van Stokkum, I. H. M. Glotaran : A Java -Based Graphical User Interface for the R Package TIMP. J. Stat. Softw. 2012, 49 (3).

(37) Striker, G.; Subramaniam, V.; Seidel, C. A. M.; Volkmer, A. Photochromicity and Fluorescence Lifetimes of Green Fluorescent Protein. J. Phys. Chem. B 1999, 103 (40), 8612-8617.

(38) Legge, F. S.; Nyberg, G. L.; Peel, J. B. DFT Calculations for Cu-, Ag-, and AuContaining Molecules. J. Phys. Chem. A 2001, 105 (33), 7905-7916.

(39) Check, C. E.; Faust, T. O.; Bailey, J. M.; Wright, B. J.; Gilbert, T. M.; Sunderlin, L. S. Addition of Polarization and Diffuse Functions to the LANL2DZ Basis Set for PBlock Elements. J. Phys. Chem. A 2001, 105 (34), 8111-8116.

(40) Pyykkö, P.; Mendizabal, F. Theory of $d^{10}-d^{10}$ Closed-Shell Attraction. III. Rings. Inorg. Chem. 1998, 37 (12), 3018-3025.

(41) Bu, M.; Reimann, C.; Pantazis, D. A.; Bredow, T.; Neese, F. Geometries of ThirdRow Transition-Metal Complexes from Density-Functional Theory Geometries of 
Third-Row Transition-Metal Complexes from Density-Functional Theory. Society 2008, 1449-1459.

(42) Adamo, C.; Barone, V. Inexpensive and Accurate Predictions of Optical Excitations in Transition-Metal Complexes: The TDDFT/PBE0 Route. Theor. Chem. Acc. 2000, 105 (2), 169-172.

(43) Kang, R.; Chen, H.; Shaik, S.; Yao, J. Assessment of Theoretical Methods for Complexes of Gold(I) and Gold(III) with Unsaturated Aliphatic Hydrocarbon: Which Density Functional Should We Choose? J. Chem. Theory Comput. 2011, 7 (12), 4002-4011.

(44) Pinto, A.; Svahn, N.; Lima, J. C.; Rodríguez, L. Aggregation Induced Emission of Gold(I) Complexes in Water or Water Mixtures. Dalt. Trans. 2017, 46 (34), 1112511139.

(45) Giestas, L.; Gavara, R.; Aguiló, E.; Svahn, N.; Lima, J. C.; Rodríguez, L. Modulation of Supramolecular Gold(I) Aggregates by Anion's Interaction.

Supramol. Chem. 2018, 30 (4), 278-285.

(46) Gavara, R.; Lima, J. C.; Rodríguez, L. Effect of Solvent Polarity on the Spectroscopic Properties of an Alkynyl Gold(I) Gelator. The Particular Case of Water. Photochem. Photobiol. Sci. 2016, 15 (5), 635-643.

(47) DeRosa, M. C.; Crutchley, R. J. Photosensitized Singlet Oxygen and Its Applications. Coord. Chem. Rev. 2002, 233-234, 351-371.

(48) Buntinx, G.; Naskrecki, R.; Poizat, O. Subpicosecond Transient Absorption Analysis of the Photophysics of 2,2'-Bipyridine and 4,4'-Bipyridine in Solution. J. Phys. Chem. 1996, 100 (50), 19380-19388.

(49) Huang, R.; Avó, J.; Northey, T.; Chaning-Pearce, E.; dos Santos, P. L.; Ward, J. S.; Data, P.; Etherington, M. K.; Fox, M. A.; Penfold, T. J.; et al. The Contributions of Molecular Vibrations and Higher Triplet Levels to the Intersystem Crossing Mechanism in Metal-Free Organic Emitters. J. Mater. Chem. C 2017, 5 (25), 62696280.

(50) Ottolenghi, M.; Goldschmidt, C. R.; Potashnik, R. Intersystem Crossing in the Charge-Transfer Quenching of Molecular Fluorescence. J. Phys. Chem. 1971, 75 (8), 1025-1031. 
(51) Dias, F. B.; Bourdakos, K. N.; Jankus, V.; Moss, K. C.; Kamtekar, K. T.; Bhalla, V.; Santos, J.; Bryce, M. R.; Monkman, A. P. Triplet Harvesting with 100\% Efficiency by Way of Thermally Activated Delayed Fluorescence in Charge Transfer OLED Emitters. Adv. Mater. 2013, 25 (27), 3707-3714. 


\section{Table of Contents (TOC)}

\section{Fast intersystem crossing lead to moderate triplet yields in}

\section{gold(I) polypyridyl complexes}

Elisabet Aguiló, Artur J. Moro, João Pina, Daniela Sarmento, J. Sérgio Seixas de Melo, Laura Rodríguez, and João Carlos Lima.
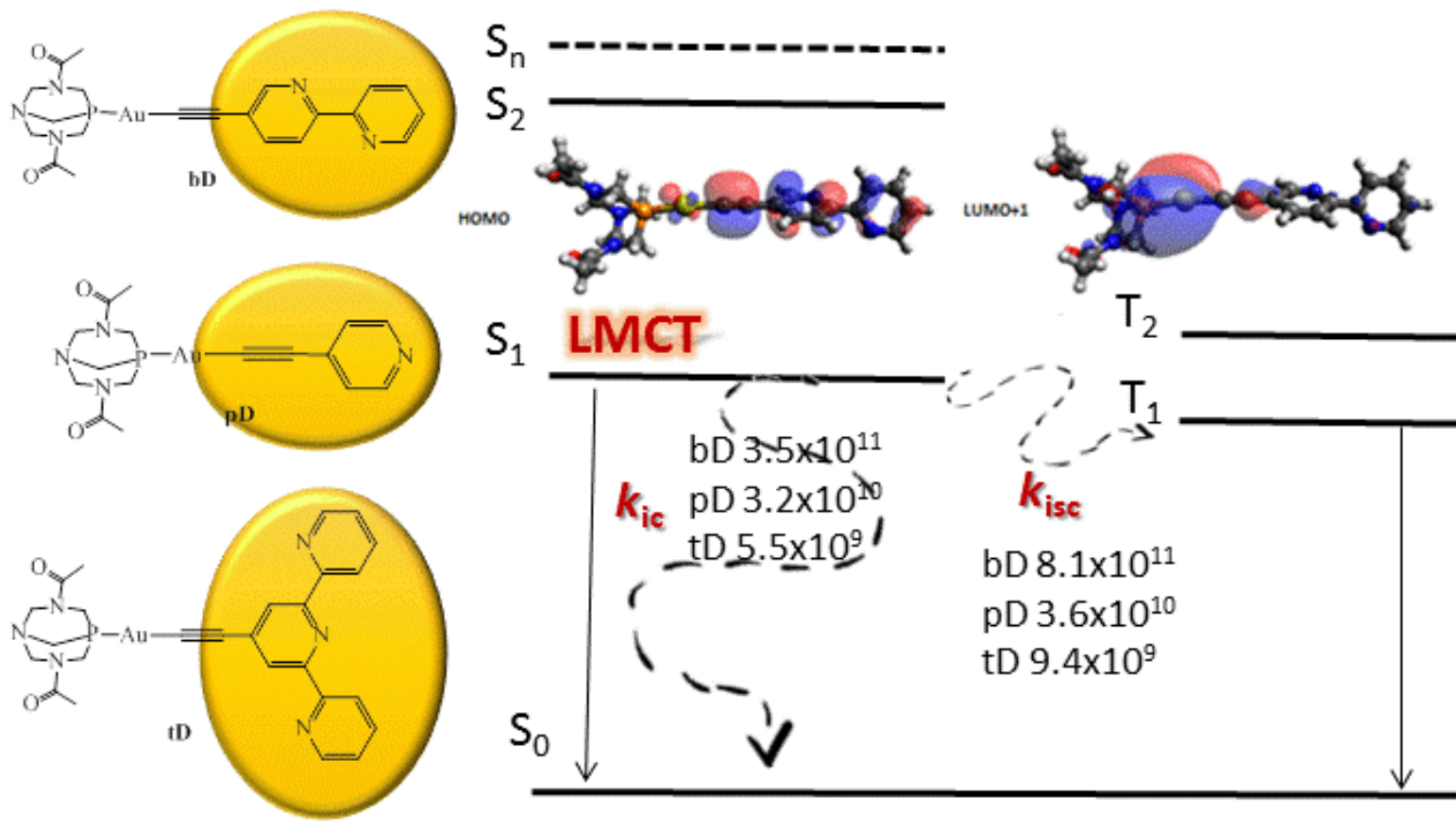

$\mathrm{S}_{1}$
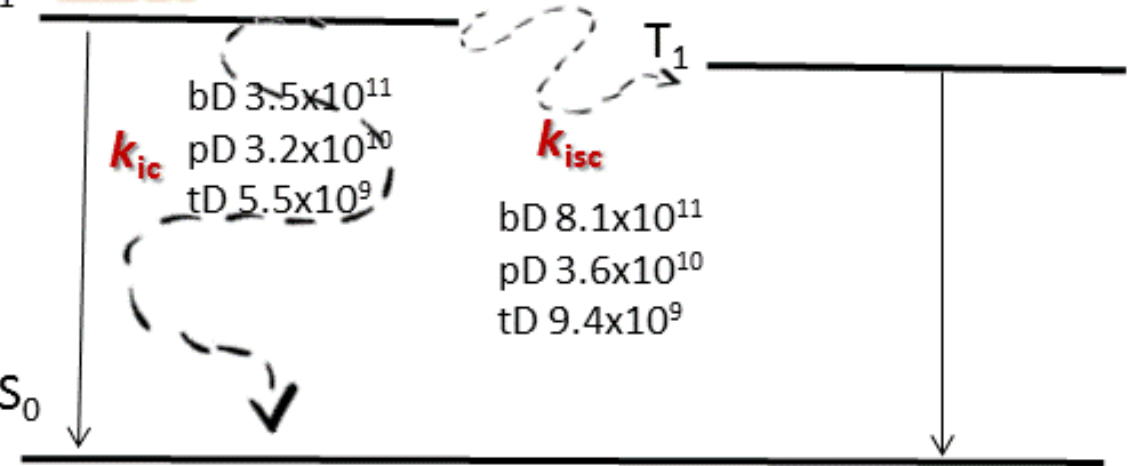

A complete photophysical characterization of a series of Gold(I) organometallic complexes bearing axial pyridyl ligands is herein presented. Results showed that triplet quantum yields do not increase with the same extent as inter-system crossing rates, due to the simultaneous increase of internal conversion. 